\title{
A Micromechanical Model of Additively Manufactured Aluminum Alloys
}

\author{
Evgeniya Emelianova $^{1,2, *}$, Varvara Romanova $^{1}$, Olga Zinovieva $^{3}$, Ruslan Balokhonov ${ }^{1}$, \\ Aleksandr Zinoviev ${ }^{3}$, and Maksim Sergeev ${ }^{1,2}$ \\ ${ }^{1}$ Institute of Strength Physics and Materials Science of SB RAS, Laboratory of Mechanics of \\ Heterogeneous Media, 634055 Tomsk, Russia \\ ${ }^{2}$ National Research Tomsk State University, Faculty of Physics and Engineering, 634050 Tomsk, Russia \\ ${ }^{3}$ University of Bremen, ISEMP, 28359 Bremen, Germany
}

\begin{abstract}
A micromechanical model is developed to predict the deformation behavior of additively manufactured aluminum alloys. Threedimensional models of grain structures typical for different microregions of the melt pool are generated by the step-by-step packing method. A crystal plasticity-based constitutive model accounting for the elastic-plastic anisotropy of face-centered cubic crystals is employed to simulate the microscale deformation in an additively manufactured aluminum alloy under loading. The grain shape and texture effects on the plastic strain localization patterns are analyzed.
\end{abstract}

\section{Introduction}

The rapid and repeated melting and solidification inherent in metal additive manufacturing (AM) result in the formation of a complex hierarchical microstructure much different from that of the cast or wrought alloys of the same composition. Understanding the mechanical behavior of AM materials and predicting their mechanical properties demand a modeling strategy that treats characteristic microstructural features and addresses deformation processes developing at various scales, with an estimation of their impact on the macroscopic stress-strain response. In this paper, we describe the mechanical behavior of an AM aluminum alloy within a microstructure-property paradigm of materials science, treating physical mechanisms of microscale plastic deformation, on the one hand, and morphological features of the grain structure, on the other hand.

\section{Microstructure-based constitutive model}

A polycrystal is considered as a conglomerate of grains with different orientations. The first step in the development of a microstructure-based mechanical model of an AM aluminum alloy involves the design of three-dimensional (3D) grain structures typical for different regions of the melt pool (Fig. 1a). For this purpose, we adopted a step-by-step packing (SSP) method [1] whose main idea is to incrementally fill a pre-meshed volume by the

\footnotetext{
*Corresponding author: emelianova@ispms.tsc.ru
} 
microstructural elements following growth functions defined analytically. The computational volume is initialized with a certain distribution of nuclei being centers of growing microstructural elements (grains). Applying different combinations of the growth functions and nucleus distributions enables the fast generation of material structures with specified morphological characteristics [1-4].

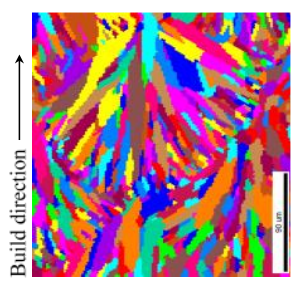

a

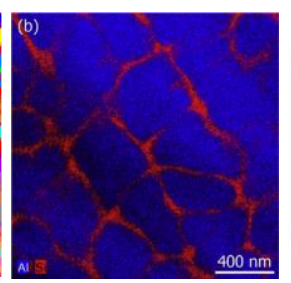

b

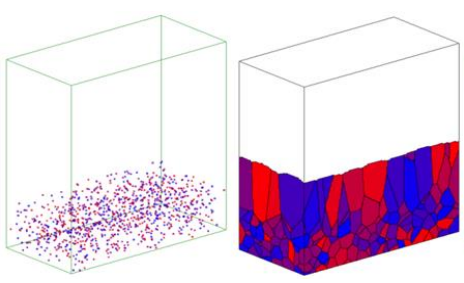

C

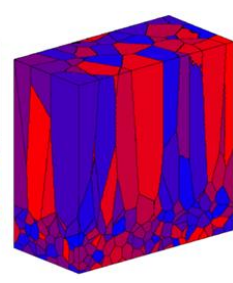

: (a) EBSD,

(b) Al and Si X-ray map [5], - and (c) steps of SSP-design of an AM microstructure

Constitutive equations to describe the mechanical behavior of grains are written using the crystal plasticity theory to take into account the elastic-plastic anisotropy and the dislocation mechanisms of plastic deformation on the grain scale. An Al-single crystal has a face-centered cubic (fcc) lattice defined here using the [100], [010], [001] crystal coordinate system. The constitutive equations in the rate form of the generalized Hooke's law for anisotropic cubic crystals characterized by three independent elastic moduli are formulated for each grain relative to its coordinate system

$$
\dot{\sigma}_{i j}=C_{i j k l}\left(\dot{\varepsilon}_{k l}-\dot{\varepsilon}_{k l}^{p}\right)
$$

Here $\sigma_{i j}$ are the components of the stress tensor, $\varepsilon_{i j}$ and $\dot{\varepsilon}_{i j}^{p}$ are the total and plastic strains, $C_{i j k l}$ is the tensor of elastic moduli; the upper dot denotes time derivative. The total strains in Eq. (1) are geometrically related to the displacement vector while plastic strains should be described using physically-based models. Assuming the validity of the Schmid's law, we define the plastic strains as a summary slip over all the active slip systems

$$
\dot{\varepsilon}_{i j}^{p}=\frac{1}{2} \sum_{\alpha} \dot{\gamma}^{(\alpha)}\left(\mathbf{s}_{i}^{(\alpha)} \mathbf{m}_{j}^{(\alpha)}+\mathbf{s}_{j}^{(\alpha)} \mathbf{m}_{i}^{(\alpha)}\right),
$$

where $\mathbf{s}_{i}^{(\alpha)}$ and $\mathbf{m}_{i}^{(\alpha)}$ are the slip direction and slip plane normal vectors for an $\alpha$ slip system. The shear strain rate $\dot{\gamma}^{(\alpha)}$ in Eq. (2) is defined by the power law [4]

$$
\dot{\gamma}^{(\alpha)}=\dot{\gamma}_{0}\left|\frac{\tau^{(\alpha)}}{\tau_{C R S S}^{(\alpha)}}\right|^{v} \operatorname{sign}\left(\tau^{(\alpha)}\right),
$$

where $\tau^{(\alpha)}$ is the resolved shear stress acting in the slip system $\alpha, \tau_{\text {CRSS }}^{(\alpha)}$ is the critical resolved shear stress (CRSS) necessary to activate slip in $\alpha, \dot{\gamma}_{0}$ is the reference slip rate, and $V$ is the strain rate sensitivity coefficient. A phenomenological function is used to describe the CRSS taking into account the cell- and grain-boundary strengthening and strain hardening

$$
\tau_{C R S S}^{(\alpha)}=\tau_{0}(d)+\tau_{g}(D)+f\left(\varepsilon_{e q}^{p}\right)
$$


The first term in the right part of Eq. (4) is the CRSS of a single crystal, depending on the average size of dendrite cells $d$ (Fig. 1b). The second term of the sum is the increase in the shear resistance according to the Hall-Petch relation, where $\mathrm{D}$ is the average grain size. The third term takes into account the strain hardening as a function of the accumulated plastic strain

$$
f\left(\varepsilon_{e q}^{p}\right)=a_{1}\left(1-\exp \left(-\varepsilon_{e q}^{p} / a_{2}\right)\right)+b_{1}\left(1-\exp \left(-\varepsilon_{e q}^{p} / b_{2}\right)\right),
$$

where $a_{1}=73 \mathrm{MPa}, a_{2}=0.07, b_{1}=16 \mathrm{MPa}, b_{2}=0.02$.

The 3D polycrystalline constitutive models were imported into the finite-element package ABAQUS/Explicit. For reduction of computational costs, the quasistatic problem was solved using the dynamic approach involving a solution of the motion equations rather than the equilibrium ones [6, 7]. A series of test calculations was conducted in [7] to meet the conditions ensuring a close agreement between the dynamic and static solutions and to check the mesh convergence of the numerical solution. In order to calibrate model parameters, a series of test calculations has been performed for single- and polycrystals using experimental data $[5,8]$.

\section{Results}

The texture at the polycrystal scale arises from two contributions. One of them is the morphological texture, i.e., a preferred shape orientation of elongated grains (Fig. 1a); another one comes from a preferred crystallographic orientation of grains (the crystallographic texture). We conducted a parametric study to reveal the influence of these contributions separately on the mechanical behavior of polycrystals under uniaxial tension. The effects of the mechanical texture were studied on polycrystalline models with elongated grains loaded along and across the long axes of crystallites. In order to exclude the influence of the crystallographic texture, the grain orientations were specified by randomly varying Euler angles over the entire range of possible values. To study the combined effect of the mechanical and crystallographic textures, we set cube textures of different strength in specimens with elongated grains. Similarly, for equiaxed polycrystals, simulations were performed for a non-textured material and specimens with cube textures of different strength. The texture strength was varied by allowing the crystal coordinate systems of different grains to deviate from the position corresponding to the cube texture by 5,10 , and $20^{\circ}$.

The crystallographic texture is found to significantly affect the localization of plastic strains. As an example, plastic strain fields in textured and non-textured polycrystals with equiaxed and elongated grains are shown in Fig. 2. For non-textured polycrystals, plastic deformation is initially localized near the grain boundaries which serve as barriers to slip propagation and as sources of stress concentration at the microscale. On further loading, larger-scale areas of plastic strain localization are formed that have a non-crystallographic nature. These mesoscale bands pass perpendicular to the tensile direction through the entire surface regardless of the grain crystallographic orientations. The stronger the texture, the more the material behavior approaches to the behavior of a single crystal. In the textured polycrystals, the grain boundaries practically do not contribute to the strain localization resistance, while stress concentration is observed only in single regions near grain boundaries. The mesoscale shear bands that pass through entire groups of grains form already at the initial stage of plastic deformation. In the case of the textured material, the grain shape has only a minor effect on the strain localization pattern. However, for nontextured polycrystals, the shape of grains is a significant factor influencing plastic strain 
localization. For polycrystals with elongated grains loaded along and across the long axes of crystallites, the anisotropy of mechanical properties is most pronounced at the initial stage of plastic deformation, where the grain boundaries are the primary sources of stress concentration and plastic strain localization.
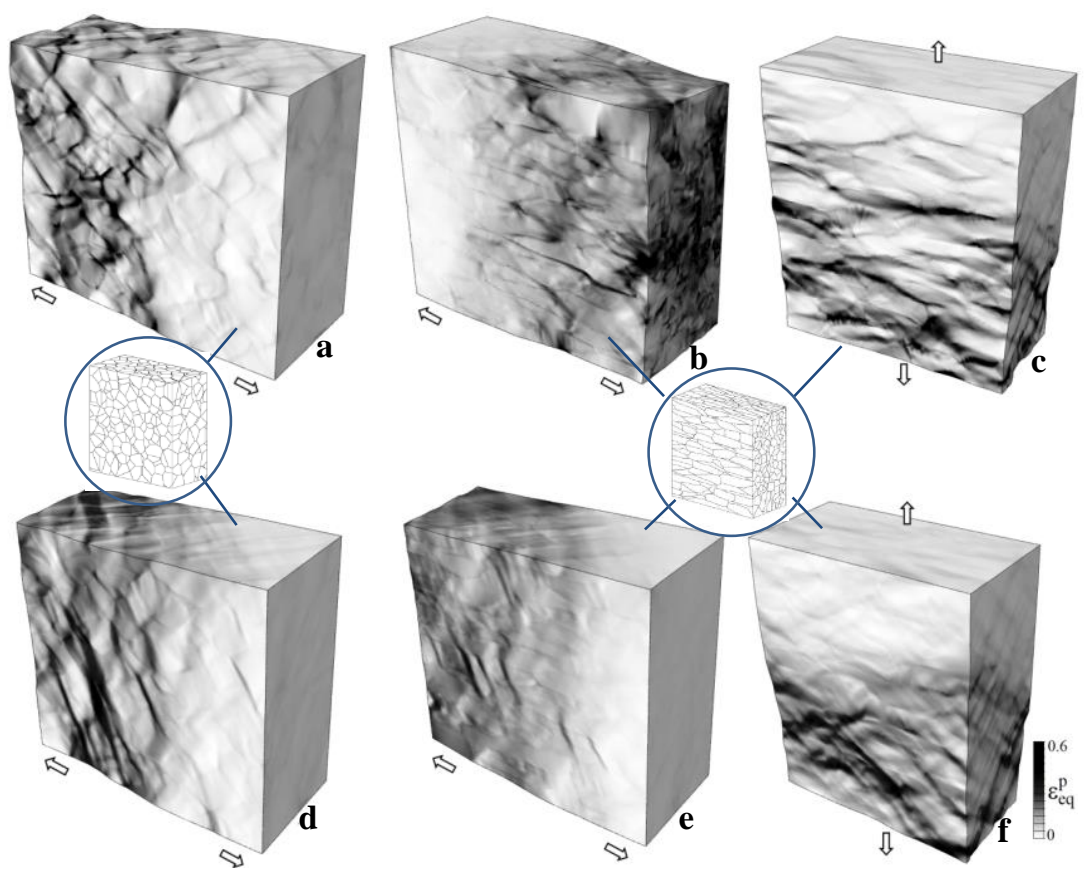

Fig. 2. Equivalent plastic strain fields in model polycrystals with equiaxed and elongated grains: (a-c) no texture, (d-f) moderate cube texture; $\varepsilon=0.06$.

This work is supported by the Deutsche Forschungsgemeinschaft (Grant No. PL 584/4-1) and the Russian Foundation for Basic Research (Grant No. 18-501-12020).

\section{References}

1. V. Romanova, R. Balokhonov, Engng with Comput. (2019)

2. R.R. Balokhonov, V.A. Romanova, S.A. Martynov, A.V. Zinoviev, O.S. Zinovieva, E.E. Batukhtina, Comput. Mater. Sci. 116 (2016)

3. V.A. Romanova, R.R. Balokhonov, O.S. Emelyanova, Phys. Mesomech. 14, 3-4 (2011)

4. R. Balokhonov, V. Romanova, E. Batukhtina, M. Sergeev, E. Emelianova, Facta Univ., Ser. Mech. Eng. 16, 1 (2018)

5. J. Wu, X.Q. Wang, W. Wang, M.M. Attallah, M.H. Loretto Acta Mater. 117 (2016)

6. V. Romanova, R. Balokhonov, E. Emelianova, O.Zinovieva, A. Zinoviev, Facta Univ., Ser. Mech. Eng. 17, 2 (2019)

7. V. Romanova, R. Balokhonov, E. Batukhtina, E. Emelianova, M. Sergeev, Phys. Mesomech. 22, 4 (2019)

8. L. Thijs, K. Kempen, J.-P. Kruth, J.V. Humbeeck, Acta Mater. 61, (2013) 